\title{
Computational Fluid Dynamics using OpenCL - a Practical Introduction
}

\author{
$\underline{\text { T Bednarz }^{1}}$, Luke Domanski ${ }^{2}$ and J A Taylor $^{3}$ \\ ${ }^{l}$ CSIRO Mathematics Informatics \& Statistics, Sydney, Australia. \\ Email: tomasz.bednarz@,csiro.au) \\ 2 CSIRO Advanced Scientific Computing, Sydney, Australia \\ 3 CSIRO Mathematics Informatics \& Statistics, Canberra, Australia
}

\begin{abstract}
The main aim of the Computational Fluid Dynamics (CFD) simulations is to reconstruct the reality of fluid motion and behaviour as accurately as possible in order to better understand the natural phenomena under specified conditions. Ideally, general solutions can also cover different scales and geometric configurations. Unfortunately, due to expensive algorithms, classic CFD codes most often require long computational times to satisfy the convergence criteria. With the advent of high-performance GPUs with massively-parallel multi-threaded architectures, basic CFD algorithms can now be implemented to give results in near real-time. The current work will briefly review our existing explicit solver based on finite difference methods, the derivation and discretisation of the mathematical model and equations, through to GPU algorithm implementation. During presentation, several case studies computed using CSIRO's CPU/GPU supercomputer cluster will be described and compared against well known analytical and experimental solutions, i.e. natural convection, driven cavity, scaling analysis, magneto-thermal convection, etc.
\end{abstract}

Keywords: $\quad$ Computational Fluid Dynamics, Simulations, Numerical Modelling 


\section{INTRODUCTION AND MODEL EQUATIONS}

Experiments with fluids are usually very expensive and many times not feasible, or require work in harmful environments, etc. Therefore, in such cases, we're trying to replace them with numerical modelling whenever possible. Here comes Computational Fluid Dynamics (CFD) that aims to reconstruct the reality of fluid motion and behaviour as accurately as possible in order to better understand the natural phenomena under specified conditions. Ideally, general solutions can also cover different scales and geometric configurations and should be with an agreement with equivalent experimental results.

Unfortunately, due to expensive algorithms, classic CFD codes most often require long computational times to satisfy the convergence criteria. With the advent of high-performance GPUs with massively-parallel multi-threaded architectures, basic CFD algorithms can now be implemented to give results in near realtime. The algorithms used to solve these problems utilize GPU and in this case OpenCL. Presented results demonstrate that GPU can be successfully used to accelerate fluid simulations. We have seen significant gains in productivity and opportunity as a result of leveraging GPUs, being able to tackle computational problems in which execution time was previously infeasible.

\subsection{Model equations}

In order to solve general incompressible thermo-fluids problems in Cartesian coordinate system, the NavierStokes and energy equations can be defined as follows:

$$
\begin{aligned}
& \vec{\nabla} \cdot \vec{U}=0 \\
& \frac{\mathrm{D} \vec{U}}{\mathrm{D} t}=-C_{1} \vec{\nabla} P+C_{2} \nabla^{2} \vec{U}+C_{3}\left(\theta-C_{4}\right) \\
& \frac{\mathrm{D} \theta}{\mathrm{D} t}=C_{5} \nabla^{2} \theta
\end{aligned}
$$

where $\vec{U}$ is the velocity, $t$ is the time, $P$ is the pressure, $\theta$ is the temperature and coefficients $C_{1}$ to $C_{5}$ will depend on a specific case considered. The same methodology can be applied to simulate wide range of different fluid flows: pipe flows, force convections, magneto-thermal convection, scaling analysis, exchange flows in reservoir model, mixing, fountain flows, bubble flow, step flows, heat exchangers, ventilation problems, etc. For instance, if under the consideration is a square cavity heated from one vertical wall and cooled from the opposite one with top and bottom walls kept adiabatic, those coefficients for dimensionless solution are defined as follows:

$$
C_{1}=1 ; C_{2}=\operatorname{Pr}=\frac{v}{\alpha} ; C_{3}=\operatorname{Pr} \cdot \operatorname{Ra}=\frac{v}{\alpha} \cdot \frac{g \beta(\Delta \theta) l^{3}}{\alpha v} ; C_{4}=0 ; C_{5}=1.0
$$

where $v$ is the kinematic viscosity, $\alpha$ is the thermal diffusivity, $g$ is the gravitational acceleration, $\beta$ is the thermal expansion coefficient, $\Delta \theta$ is the maximum temperature difference, $l$ is the length of the cube, $\operatorname{Pr}$ is the Prandl number describing ratio of momentum and thermal diffusivities and $R a$ is the Rayleigh number associated with buoyancy driven flow describing strength of the convection.

\section{NUMERICAL APPROACH}

Those equations are approximated with finite difference equations and the HSMAC (Highly Simplified Marker and Cell) method (Bednarz et al. 2005-2010, Hirt 1975) is used to iterate mutually the pressure and velocity fields on staggered mesh/grid allocation system, see Figure 1. The inertial terms in momentum equations are approximated using a third-order upwind UTOPIA scheme (Tagawa 1996). The absolute convergence criteria for the numerical solutions are specified based on the residual sums of all conserved quantities. If the residual sum is less than $10^{-6}$ for each conserved quantity, the equations are deemed to have converged at a specific time step. The time-step is chosen to ensure numerical stability according to the CFL condition. The numerical methods used in this work for simulation of natural convection have been widely verified by co-workers, by both the numerical and the experimental investigations for closely related problems (Bednarz et al. 2005-2010). 

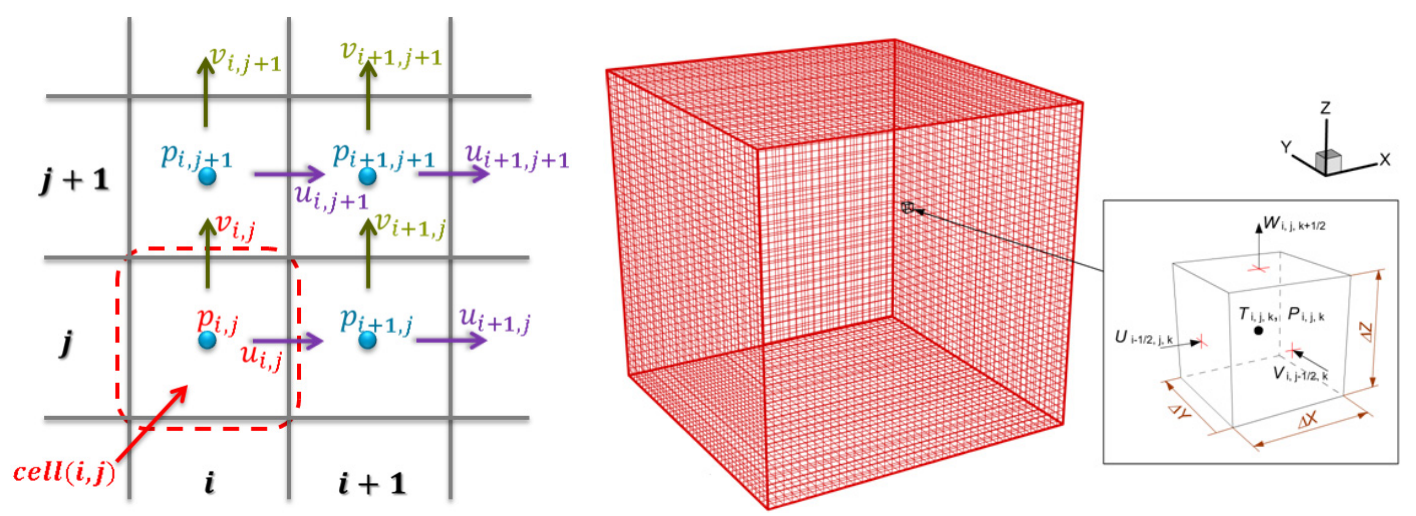

Figure 1. Staggered mesh grid allocations prevents possible pressure oscillations. Left: for 2-D cases all scalar variables are defined in the centre of cells, and vector variables in at the centre of vertical and horizontal cell faces accordingly. Right: grid for 3-D cases.

\section{OPENCL IMPLEMENTATION}

The numerical code is ported from CPU to GPU version using the OpenCL API, see references. This was motivated by the need of improving computation speed, as in some cases, e.g. computation of single case of boundary layer evolution (Bednarz et al. 2009) could take up to 12 hours on grid size 256x256. Therefore, all critical parts of previously available CPU code are re-implemented in several OpenCL kernels that could be executed by thousands simultaneous threads by a GPU. Figure 2 shows the flow chart of our HSMACOpenCL solver. As seen, the initialization part includes: reading initial configuration files describing geometry and parameters of the problem to be computed, allocating memory for all field variables (pressure, velocity components, temperatures and spatial coordinates), preparing boundary conditions flags (to mark regions where the boundaries are located and what is their type). Once that's done, the OpenCL is initialized, proper compute device is attached to its context and the CL program is compiled. Also the device memory buffers are created and filled with initial data.

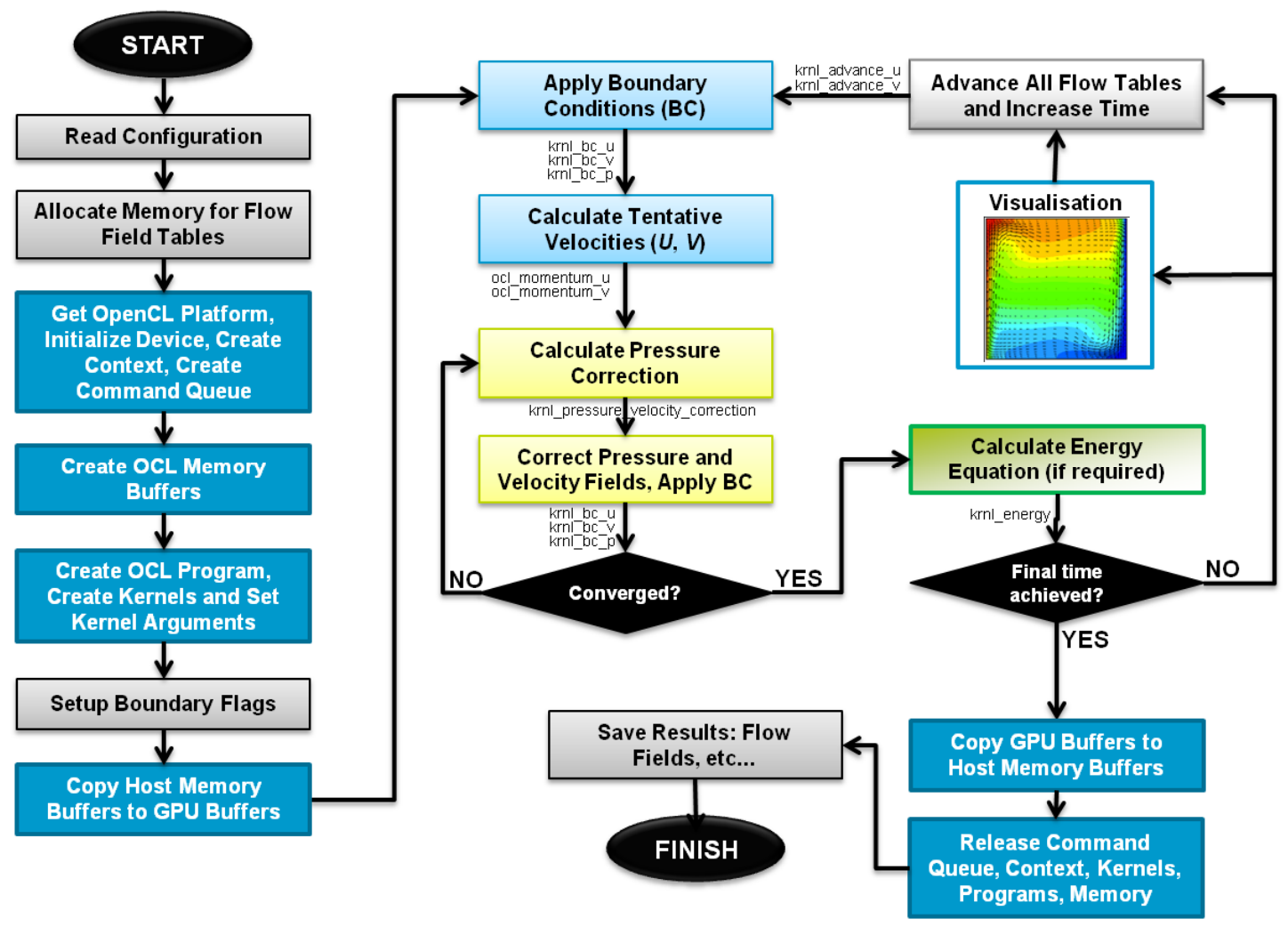

Figure 2. Flow char of the OpenCL solver. 
Bednarz et al., Computational Fluid Dynamics using OpenCL - a Practical Introduction

Bednarz, T., Caris, C., and Taylor, J. (2010) A practical introduction to Computational Fluid Dynamics on GPUs, GTC 2010, San Jose, USA, September 20-23.

Botella, O., and Peyret, R. (1998) Benchmark spectral results on the lid-driven cavity flow, Computers \& Fluids, Volume 27, Issue 4, Pages 421-433.

CSIRO GPU cluster: http://www.csiro.au/resources/GPU-cluster.html.

De Vahl Davis, G. (1983) Natural convection of air in a square cavity: a bench mark numerical solution, International Journal of Numerical Methods in Fluids, Vol 3, pp 249-264.

Hirt, C.W., Nichols, B.D., and Romero, N. (1975) A numerical solution algorithm for transient fluid flow, Los Alamos Scientific Laboratory, LA-5852.

OpenCL: http://www.khronos.org/opencl/

Tagawa, T., and Ozoe, H. (1996) Effect of Prandtl number and computational schemes on the oscillatory natural convection in an enclosure, Numerical Heat Transfer A 30, 271-282.

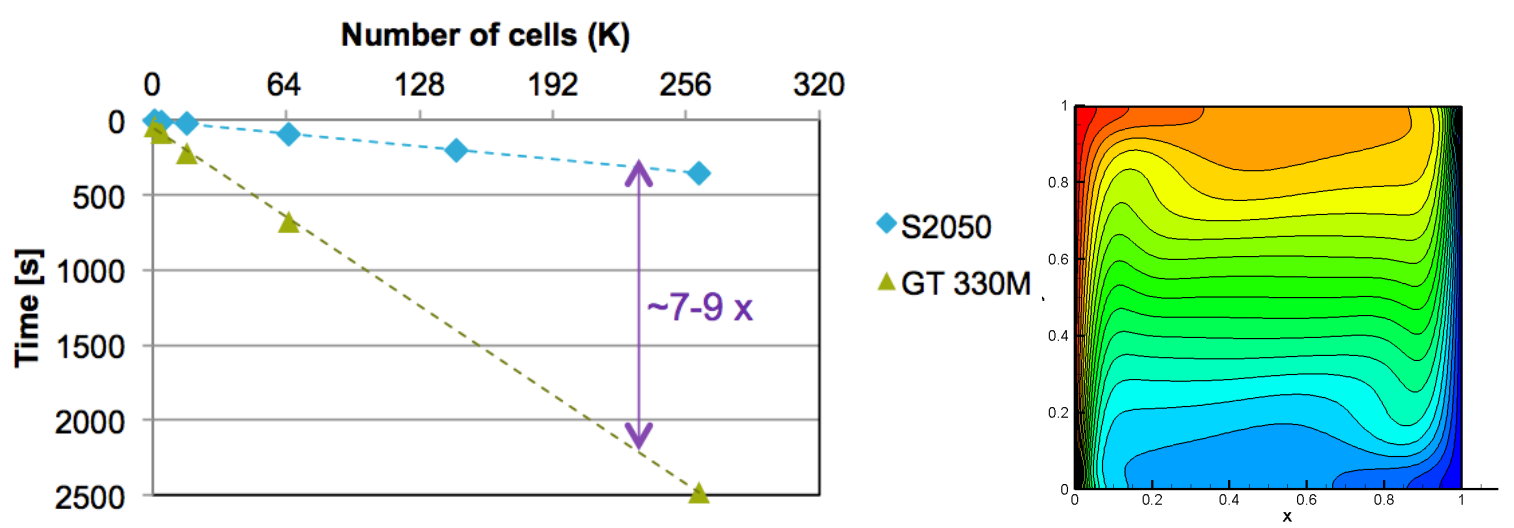

Figure 4. Total execution times for S2050 and GT330M against number of computational cells and converged temperature contours for $\operatorname{Pr}=0.71$ and $R a=10^{6}($ left wall $=$ heated, right wall $=$ cooled). 